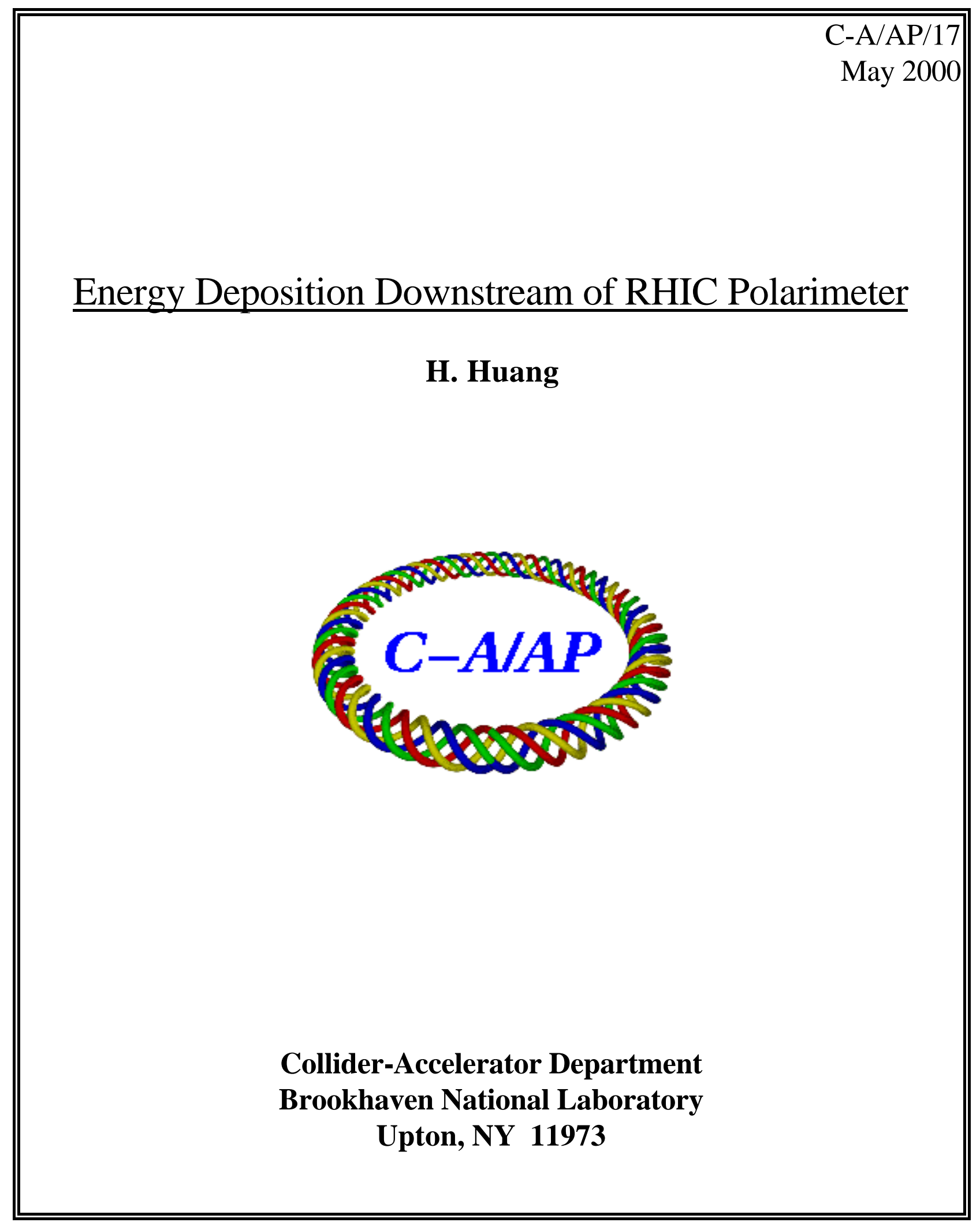




\title{
Energy Deposition Downstream of RHIC Polarimeter
}

\author{
Haixin Huang
}

\begin{abstract}
The RHIC polarimeter sits between Q3 and Q4 and the target is very close to Q4. This notes addresses the issue of possibility of quench of Q4. The results shows that with the given conditions, it is likely that Q4 can survive the scattering particle shower without quench. Nevertheless, the target frame will be evaporated in some cases.
\end{abstract}

The polarimeter target in blue ring of RHIC is about 5 meter upstream of Q4. If the beam hit the target frame by accident, it is a concern that the Q4 may quench due to the large amount of scattering particles from the target frame. This note is trying to scale the simulation done for scraper and get some rough estimate on this issue.

Some simulation has been done with CASIM for scraper ${ }^{[1]-[2]}$. The simulations show that one of the main contribution to the Q4 heating is so-called "suck-in electrons" (electrons are sucked into the quadrupole due to their relative light mass). The location of maximum energy density was at the downstream end of Q4. Since in both cases the interaction length is larger than the material thickness, the results can be scaled to the case when protons hit the frame. The simulation shows that for $100 \mathrm{GeV} / \mathrm{c}$ Gold beam and Q4 at $35 \mathrm{~m}$ away from scraper, the energy densities is $d E_{i}(p=100 \mathrm{GeV} / c, R=35 \mathrm{~m})=25.1 \pm 4.5 \times 10^{-3} \mathrm{GeV} / \mathrm{cm}^{-3}$ per ion interaction. To scale this number for proton beam at $250 \mathrm{GeV} / \mathrm{c}$, we need to make a few assumptions:

1)assuming the energy deposition density is proportional to the beam energy;

2)assuming the energy density is proportional to $1 / R^{2}$, where $R$ is the distance from scattering center;

3)assuming the average density of the coil material is $6 \mathrm{~g} / \mathrm{cm}^{3}$;

4)assuming the critical temperature $T_{c}$ of the super-conductor is inversely proportional to the magnetic field (and beam energy) ${ }^{[3]}$, consequently, the tolerable energy deposit in the coil will be higher at lower magnetic fields.

The FNAL quench threshold was experimentally determined as $2 \mathrm{~mJ} / \mathrm{g}$ for instantaneous loss, and $8 \mathrm{~mW} / \mathrm{g}$ for beam loss over times in the 1-10 second range ${ }^{[1]}$. 
Table 1

\begin{tabular}{|l|l|l|}
\hline beam energy & distance & intensity \\
\hline $25 \mathrm{GeV}$ & $5 \mathrm{~m}$ & $3.79 \times 10^{14}$ \\
\hline $50 \mathrm{GeV}$ & $5 \mathrm{~m}$ & $9.47 \times 10^{13}$ \\
\hline $100 \mathrm{GeV}$ & $5 \mathrm{~m}$ & $2.37 \times 10^{13}$ \\
\hline $250 \mathrm{GeV}$ & $5 \mathrm{~m}$ & $3.79 \times 10^{12}$ \\
\hline $250 \mathrm{GeV}$ & $35 \mathrm{~m}$ & $1.86 \times 10^{14}$ \\
\hline
\end{tabular}

Table 1: Intensity threshold to quench Q4 or Q3.

These values were obtained at FNAL with magnets operating at $90 \%$ of short sample. Since the RHIC quads operate as $60 \%$ of short sample, this threshold can be raised as $6 \mathrm{~mJ} / \mathrm{g}^{[4]}$. One can deduce the corresponding threshold for RHIC with proton beams from these numbers. Given above assumptions, the energy deposition density in the end of Q4 per proton interaction is given by

$$
d E_{p}(p, R)=\frac{d E_{i}}{A} \cdot \frac{p}{100} \cdot\left(\frac{35 m}{R}\right)^{2} \cdot \frac{1}{\rho_{m}}
$$

where $A=197$ is the atomic number for gold ion beam, $d E_{i}$ is energy density generated for gold beam, $p$ is the beam momentum, $R$ is the distance from the scattering center to the end of Q4 and $\rho_{m}$ is the average density of the coil material. Assuming that particles interact with the target frame will be lost in one turn, the total beam intensity can hit the aluminum frame without quenching Q4 is

$$
I_{t}(p, R)=\frac{6 \cdot \frac{m J}{g}}{d E_{p}(p, R)} \cdot \frac{250}{p} \cdot \frac{\lambda}{d \rho}
$$

where $\lambda$ and $\rho$ are the nuclear interaction length and density of $\mathrm{Al}, R=5 \mathrm{~m}$ is the distance from scattering center to the end of Q4 in blue ring, $d=1.5 \mathrm{~mm}$ is the thickness of the Al frame. The $250 / p$ factor assumes the "enthalpy reserve" of the super-conductor grows linearly with energy and $6 \mathrm{~mJ} / \mathrm{g}$ is referred to the top energy, i.e., $250 \mathrm{GeV}$. Table 1 lists the intensity thresholds for different energies.

The table shows that that $\mathrm{Q} 4$ will not quench below $100 \mathrm{GeV}$ even when the whole beam(full intensity is 114 bunches $\times 2 \times 10^{11}=2.28 \times 10^{13}$ ) hit the frame. Nevertheless, the target frame will be melted if hit since the melting point for aluminum is only $1000 \mathrm{~K}$. It is certainly no quench problem in yellow ring either since Q3 is 35m away. For energies higher than $50 \mathrm{GeV}$, we need to consider the geometry of the frame and beam size in more detail.

The normalized beam emittance of polarized proton beam in the AGS is 10-20 $\pi \mathrm{mm}$-mrad. One can take it as the emittance in RHIC. The beta functions at the 
target location are $\beta_{x}=21 \mathrm{~m}, \beta_{y}=29.2 \mathrm{~m}$. The target frame is $30 \mathrm{~mm} \times 30 \mathrm{~mm}$ with the carbon target located at the opening end. When beam is perfectly centered in the frame, it is $15 \mathrm{~mm}$ away from the closest edge of the frame. In the case that the beam was bumped away from the center for beam polarization profile scan, local correctors will be used to kick the beam away from the center. The maximum strength of the correctors can generate $38 \mathrm{~mm}$ move at injection energy, $25 \mathrm{GeV}{ }^{[5]}$. This number is inversely proportional to the beam energy and would be only $3.8 \mathrm{~mm}$ at top energy $250 \mathrm{GeV}$. The target frame position can be controlled within $2 \mathrm{~mm}$. The overall orbit of RHIC beam can be set with offset less than $2 \mathrm{~mm}$ (the number from 1999 commissioning). So the beam could be off the frame center by as much as $4 \mathrm{~mm}$. Given those conditions, the worst scenarios are when beam hits frame due to the maximum corrector strength with the $4 \mathrm{~mm}$ offset taking into account. If the portion of the beam hit the frame is far less than the threshold listed in Table 1, Q4 will not quench. Table 2 and 3 list the percentage of beam hit the frame at different energies.

Table 2

\begin{tabular}{|c|c|c|c|c|c|}
\hline $\begin{array}{c}\text { beam } \\
\text { energy }\end{array}$ & $\begin{array}{c}\text { beam } \\
\text { size }(\mathrm{mm})\end{array}$ & $\begin{array}{c}\text { distance } \\
\left(\sigma_{y}\right)\end{array}$ & $\begin{array}{c}\text { corrector } \\
\text { strength }(\mathrm{mm})\end{array}$ & $\begin{array}{c}\text { quench } \\
\text { intensity }\end{array}$ & $\begin{array}{c}\text { intensity } \\
\text { on target }\end{array}$ \\
\hline $25 \mathrm{GeV}$ & 1.35 & 8.1 & 38 & 16.6 & 1 \\
\hline $50 \mathrm{GeV}$ & 0.955 & 11.5 & 19 & 4.15 & 1 \\
\hline $80 \mathrm{GeV}$ & 0.754 & 14.6 & 11.9 & 1.61 & 0.82 \\
\hline $90 \mathrm{GeV}$ & 0.711 & 15.5 & 10.6 & 1.28 & 0.268 \\
\hline $100 \mathrm{GeV}$ & 0.675 & 16.3 & 9.5 & 1.04 & 0.013 \\
\hline $250 \mathrm{GeV}$ & 0.427 & 25.7 & 3.8 & 0.16 & 0 \\
\hline
\end{tabular}

Table 2: For $10 \pi \mathrm{mm}-\mathrm{mrad}$ beam. The second column is the vertical beam size $\sigma_{y}$, the third column is the distance from the beam center to the edge of the frame with the $4 \mathrm{~mm}$ offset in the unit of $\sigma_{y}$; the fourth column is the maximum movement can be generated by corrector; the fifth column is the quench intensity threshold for Q4 in unit of full intensity; and the sixth column is the amount of beam which will hit the frame with the maximum corrector strength. It is also in unit of full intensity.

A similar calculation for the ultra-thin carbon target shows that the target can stay in the full intensity beam. The quench threshold for the thin target is way above the full intensity. On the other hand, the half life time of the thin carbon target is about 6 hours when sitting in the full intensity beam. If the target is stuck in the beam, it will likely disappear after several hours.

The results show that the intensity on the target frame is likely less than the quench threshold in all cases. Please keep in mind that this is a very rough estimate. In RHIC polarized proton operation, one should run less intensity first to confirm the 
Table 3

\begin{tabular}{|c|c|c|c|c|c|}
\hline $\begin{array}{c}\text { beam } \\
\text { energy }\end{array}$ & $\begin{array}{c}\text { beam } \\
\text { size }(\mathrm{mm})\end{array}$ & $\begin{array}{c}\text { distance } \\
\left(\sigma_{y}\right)\end{array}$ & $\begin{array}{c}\text { corrector } \\
\text { strength }(\mathrm{mm})\end{array}$ & $\begin{array}{c}\text { quench } \\
\text { intensity }\end{array}$ & $\begin{array}{c}\text { intensity } \\
\text { on target }\end{array}$ \\
\hline $25 \mathrm{GeV}$ & 1.91 & 5.7 & 38 & 16.6 & 1 \\
\hline $50 \mathrm{GeV}$ & 1.35 & 8.1 & 19 & 4.15 & 1 \\
\hline $80 \mathrm{GeV}$ & 1.07 & 10.3 & 11.9 & 1.61 & 0.74 \\
\hline $90 \mathrm{GeV}$ & 1.01 & 10.9 & 10.6 & 1.28 & 0.33 \\
\hline $100 \mathrm{GeV}$ & 0.96 & 11.5 & 9.5 & 1.04 & 0.058 \\
\hline $250 \mathrm{GeV}$ & 0.60 & 18.2 & 3.8 & 0.16 & 0 \\
\hline
\end{tabular}

Table 3: For $20 \pi$ mm-mrad beam. The meaning of each column is similar to those of table 2 .

proper function of correctors and target positions, then put high intensity beam into RHIC.

The author gratefully acknowledges several helpful discussions with Alan Stevens.

\section{REFERENCES}

1. A.J. Stevens, Betatron Scraping at RHIC: General Remark and Sample Calculations, AD/RHIC/RD-45, September, 1992.

2. A. J. Stevens, Energy Deposition Downstream of the Internal Dump, AD/RHIC/RD97, December, 1995.

3. S.L. Wipf, Stability and Degradation of Super-conducting Current-Carrying Devices, LA-7275(Los Alamos National Lab), December, 1978.

4. A. J. Stevens, private communications.

5. V. Ptitsyn, private communications. 